\title{
Adaptive Short-Time Fractional Fourier Transform Used in Time-Frequency Analysis
}

\author{
Lin $\operatorname{Tian}^{12}$ \\ School of Electronics and Information, Yili Normal University, Yining, 830054, China \\ E-mail: tianlin201105010163.com
}

In order to improve the time-frequency resolutions of short-time fractional Fourier transform, adaptive short-time fractional Fourier transform (ASTFRFT) method is used in this paper. The optimal order of ASTFRFT is given by maximizing kurtosis of signals in fractional domain, where the window width of ASTFRFT is searched by the maximal Shannon entropy of timefrequency distribution. Short-time fractional Fourier transform has lots of transform orders and its window width has several options. ASTFRFT selects its fractional transform orders wtih kurtosis, and its width window with Shannon entropy of time-frequency distribution. As to nonstationary signals, the experimental results reveal that the ASTFRFT has better effect than the short-time fractional Fourier transform with arbitrary fractional orders and with arbitrary window width. For multi-components signals, ASTFRFT can easier and more efficiently select the optimal orders and their suitable window width for short-time fractional Fourier transform, which can provide good time-frequency resolutions.

CENet2017

22-23 July 2017

Shanghai, China

\footnotetext{
${ }^{1}$ Speaker

${ }^{2}$ This work is supported by the Natural Science Foundation of the Xinjiang Uygur Autonomous Region (Grant:2016D01C384)
} 


\section{Introduction}

Time frequency analysis, as a powerful tool to analyze non-stationary signals, can mapa 1D signal of time into a 2D image of frequency and time, which describes how the frequency content varies with time. There are two main types of time-frequency analysis methods: linear time frequency analysis method and nonlinear time frequency analysis method.The traditional time frequency analysis methods are short-time Fourier transform(STFT), wavelet transform, Wigner-Ville distribution (WVD) and so on. STFT is restricted by the uncertainty principle and fixed window function. Therefore, the time frequency resolution is low. Time frequency analysis results of wavelet transform depend on the choice of the mother wavelet. Using nonlinear time frequency analysis method WVD to deal with multi-components signals, WVD creates cross-terms. It is difficult to distinguish auto-terms from cross-terms for time-frequency analysis results. Smoothed WVD can suppress cross-terms, and the time-frequency resolutions decline simultaneously. The methods to improve time frequency resolution are classified into three main types: 1) adopting a new time frequency method, 2) optimizing the kernel function of smoothed WVD distribution, 3) rotating the time-frequency plane.

The mathematical tool of rotating the time-frequency plane is fractional Fourier transform (FrFT), which is a generalization of the Fourier transform and introduced to the signal processing field by Almeida (1994), who interpreted FrFT as the operator of rotating the signal in the time frequency plane for the first time [1]. FrFT combines the traditional time frequency analysis methods with several fractional time frequency analysis methods [2-5].

Capus and Brown proposed short-time fractional Fourier transform (STFRFT) used for chirp signal processing. The transform order of STFRFT has two types: global and local. Global optimal order method uses hill climbing to search the optimal order, while the local optimal order method calculates the optimal order windowed signal. [6]. Stanković et al. utilized the maximum of second-order central moment to determine the optimal transform order of STFRFT, which was used to pseudo Wigner-Ville distribution, and the fractional time-frequency distribution with high concentration was obtained [7]. Tao et al. presented that by converting the signal from time domain to fractional-time and fractional-frequency domain, this algorithm can detect chirp signal with different orders of STFRFT [8]. A fast algorithm for searching the optimal order is given, and the time-frequency resolutions of STFRFT and STFT are compared in the literature [9].Lu et al. proposed adaptive short-time fractional Fourier transform with time-frequencysegmentation for chirp signal processing [10].

The resolution of STFRFT can be effected by the window width and the fractional transform order. Traditional optimal STFT calculates instantaneous frequency first, which results in adaption of the window widths accordingly[11]. The window function is determined by the feedback of time-frequency resolution[12-13].

In this study, the method of adaptive short-time fractional Fourier transform (ASTFRFT) is applied, where the optimal order of ASTFRFT is determined by the latest method. The signal gets high concentration in fractional time-frequency domain, and with the rotation property, the 
time-frequency results are rotated to time-frequency domain. The window width is provided according to the time-frequency resolution with the feedback method.

\section{Theory}

\subsection{Short-Time Fractional Fourier Transform}

The $p$-th order FrFT of $x(t)[1]$

$$
X_{P}(u)=F^{P} x(t)=\int_{-\infty}^{\infty} x(t) \phi_{p}(t, u) d t
$$

where $\phi_{p} \quad$ is the fractional transform kernel,

$$
\begin{aligned}
& A^{P} \exp \left[j \pi\left(t^{2}+u^{2}\right) \cot (\varphi)-j \pi \operatorname{tucsc}(\varphi)\right], \varphi \neq k \pi \\
& \phi_{p}=(\delta(u-t), \varphi=2 \mathrm{k} \pi \\
& \delta(u+t), \varphi=(2 \mathrm{k}+1) \pi
\end{aligned}
$$

where $\varphi=\frac{\pi}{2} p, A^{P}=\exp \left[\frac{-j \pi \operatorname{sgn}(\sin \varphi)}{4}+\frac{j \varphi}{2}\right] / \sqrt{|\sin \varphi|}, \mathrm{j}$ is the imaginary unit, and $k$ is an integer.

Short-time Fourier transform (STFT) is a windowed Fourier transform, similar to the traditional STFT. The windowed short-time fractional Fourier transform (STFRFT) is given as $^{[8]}$

$$
\operatorname{STFRFT}(u, v)=\int_{-\infty}^{\infty} x(\tau) g(\tau-t) \phi_{p}(t, u) e^{-j v \tau} d t
$$

where $(u, v)$ is a coordinate by rotating coordinate $(t, f)$ angle $\varphi$ counterclockwise, the variable $u$ is in fractional-time domain, and $v$ is in fractional-frequency domain, and $g(t)$ is window function, the range of transform order $p$ is [-2,2].

To enable the time-frequency distribution with a clear physical meaning, the rotation is needed from the fractional time-frequency domain to time-frequency domain:

$$
\operatorname{STFRFT}(t, f)=R_{\alpha}[\operatorname{STFRFT}(u, v)]
$$

where $R_{\alpha}$ is rotation operator, $\alpha=-\varphi=\frac{-\pi}{2} p$,the rotation relationship is as follows:

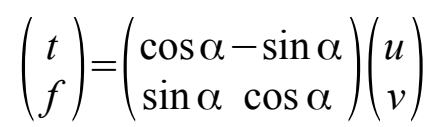

where $t, f, u, v$ represent the transformed coordinates.

\subsection{The Optimal Order Computational Method of ASTFRFT}

Generally, the signal is transformed into the best order fractional time-frequency domain to get high concentration time-frequency distribution (TFD). The best optimal order is the optimal order of fractional time-frequency domain. Kurtosis maximization approach is used to search the optimal order of fractional Gabor transform[6], Upon introdcution of the algorithm advantages for multi-component signals, the optimal order of adaptive short-time fractional Fourier transform (ASTFRFT) is generated : 


$$
p_{\text {opt }}=\arg \max \left(K_{p}\right)
$$

where $K_{p}$ is the kurtosis of $X_{P}$. The larger the coefficient of kurtosis is, the larger the steepness of the signal distribution will be. That is to say, the distribution concentration will be high. Fourier transform is a linear transformwhich cannot change the sign of kurtosis. The sign of kurtosis in fractional-time domain is a plus sign, while after the Fourier transform in fractional-frequency domain, the sign of kurtosis is still a plus sign. When the signal has maximal kurtosis in fractional-time domain where the steepness is large, the fractional timefrequency concentration is large [6].

\subsection{The Window Width Computational Method of ASTFRFT}

\subsubsection{Measurements of Concentration}

There are mainly three kinds of concentration judging criteria in time-frequency domain: Shannon entropy, energy density and kurtosis. Since the weak signal components would be ignored when adopting the coefficient kurtosis evaluating the time-frequency distributionaggregation, Shannon entropy and energy density were used as criteria. Shannon entropy to evaluate time-frequency distribution concentration is given as [14]:

$$
H=1+\sum_{m=1}^{M} \sum_{n=1}^{N} \rho(t, f) \log _{M N} \rho(t, f)
$$

The scale of $\mathrm{H}$ is $[0,1]$, when $\mathrm{H}$ is 0 , the time-frequency distribution concentration is low; when $\mathrm{H}$ is 1 , the time-frequency distribution concentration is high. For energy density, the smaller the energy density is, the higher the time-frequency distribution concentration will be. Conversely, the time-frequency distribution concentration will be lower. The energy density is expressed as:

$$
E D=\left(\sum_{m=1}^{M} \sum_{n=1}^{N} \operatorname{TFD}^{\frac{1}{q}}(t, f)\right)^{q}
$$

where the parameter $\mathrm{q}$ is the order of $E D$, the value of $\mathrm{q}$ is 3 in this study. $\mathrm{M}$ and $\mathrm{N}$ refer to the numbers of columns and rows, and TFD is time-frequency distribution.

Different time frequency analysis results in different magnitudes. To evaluate it with different methods, the time-frequency distributions should be normalized. The normalized timefrequency distribution can be expressed as:

$$
\rho(t, f)=\frac{|T F D(t, f)|^{2}}{\sum_{m=1}^{M} \sum_{n=1}^{N}|T F D(t, f)|^{2}}
$$

\subsubsection{Adaptive WindowWidth Algorithm Based on Shannon Entropy}

Gauss window has Gauss functions in time domain, frequency domain, and fractional domain[15]. It has high concentration in time domain-frequency domain.In this study, the window functions are all Gauss windows and the discrete Gauss window is expressed as

$$
g(n)=\exp \left(-0.5\left(\frac{2.5 \mathrm{n}}{K / 2}\right)^{2}\right)
$$

where $\mathrm{K}$ is the width of window function, and $\mathrm{n}$ is the sampling points of window function. If the window function width is suitable for STFRFT, the concentration of STFRFT will be high. Therefore, Shannon entropy is used to select suitable window width for ASTFRFT. The lager Shannon entropy $\mathrm{H}$ is, the better the time-frequency distribution concentration will be [11].For this reason, the largest Shannon entropy is searched for ASTFRFT. 
Algorithm 1. ASTFRFT window width searching algorithm is based on Shannon entropy.

$L$ is the window width, $L \max =\mathrm{N}, \mathrm{N}$ is the length of the signal, $L \mathrm{~min}=1$.

(1) initialization: the initial value window width $L 1, \mathrm{i}=1$;

(2) to calculate STFRFT and Shannon entropy H1 ;

(3) $\mathrm{i}=\mathrm{i}+1, \quad L_{i+1}=L_{i+1}+\Delta L, \Delta L$ is the step of window function;

(4) to calculate STFRFT and Shannon entropy $H_{i+1}$;

(5) to find the maximal Shannon entropy $H_{i}$ Hi and the corresponding $L_{i}, L_{i}$ is the optimal window width $L_{\text {opt }}$.

\subsection{The Calculating Method of ASTFRFT}

The calculating method of ASTFRFT is as follows:

(1) to calculate optimal order of signal in fractional domain by using Equation (2.1) and

(2.6);

(2) to set the initial value of window width, the searching step of window width is 1 , to calculate STFRFT by using Algorithm 1 to search the optimal window width;

(3) to calculate STFRFT in optimal order and optimal window width, and the result is ASTFRFT.

\section{Simulations Results and Analysis}

To test the advantages of ASTFRFT in improving signal time-frequency resolution, a multi-components synthetic signal is used to test its performance, and the traditional time-frequency methods are used to compared with the proposed method. The first test signal is shown in Fig. 1(a)):

$$
x_{1}(t)=4 \exp \left(i \pi\left(t^{2}+4 \mathrm{t}\right)\right) \exp \left(\frac{-\pi}{5} t^{2}\right)+\exp \left(i \pi\left(0.5 \mathrm{t}^{2}-2.5 \mathrm{t}\right)\right) \exp \left(\frac{-\pi}{9} t^{2}\right)
$$

a)

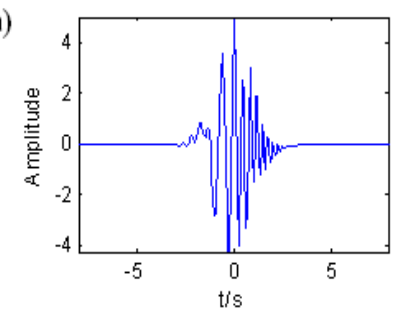

d)

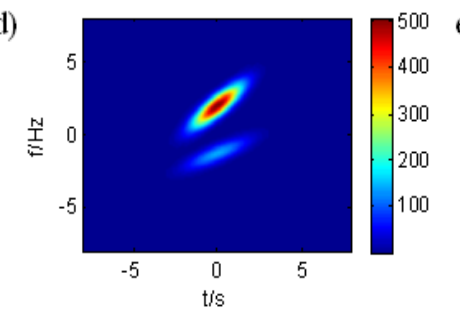

b)

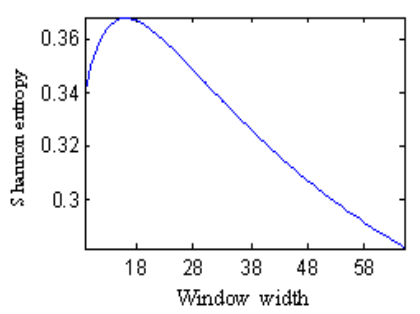

e)

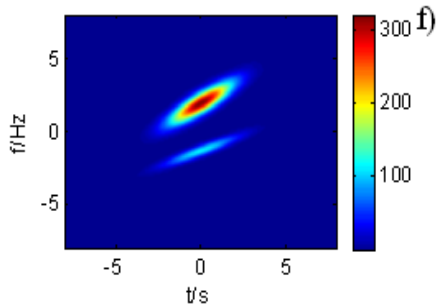

c)
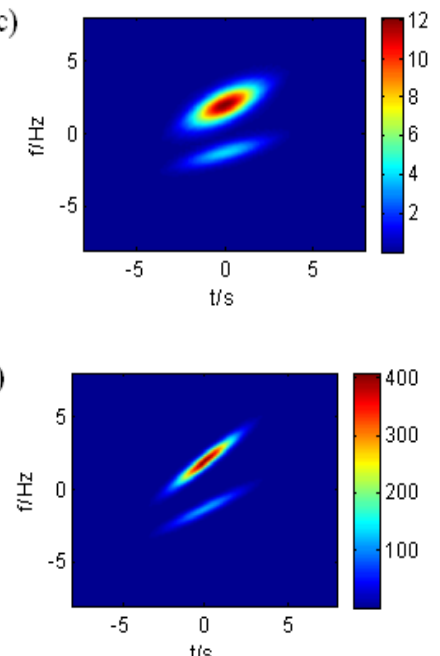

Figure 1 : Quadratic Chirp Signal and Time-Frequency Distributions, (a) the real part of $x_{1}(t)$, (b) the relationship between the Shannon entropy and the window function width, (c) STFT, (d) STFRFT with 30 points window width, (e) STFRFT with the transform order is 1.2, (f) ASTFRFT 
The signal $\quad x_{1}(t)$ is composed of two attenuation quadratic linear chirp signals. When sampling period is $0.0625 \mathrm{~s}$, sampling frequency is $16 \mathrm{~Hz}$ and $t \in[-8,8] \mathrm{s}$, sampling signal point number is 256 .

The optimal order of FrFT is 1.41 given by kurtosis maximization method and the optimal window width is 15 given by Algorithm1 for test signal. Shannon entropy with window width from 8 to 64 is shown in Fig. 1(b). Fig. 1 (c) is STFT, the algorithm of which is given to timefrequency toolbox in the website (http: // tftb. nongnu. Org/). When the window width is $1 / 4$ length of signalwhich is 256, the window width is 64. Figure 1(f) is the result of ASTFRFT. When the order equals to the optimal order, and the window width is 30. The STFRFT is shown in Fig. 1(d). When the widow width selects the optimal window width andthe transform order of FrFT with the arbitrary value, say 1.2, the according STFRFT is shown in Fig. 1(e).

Figure 1(c)-(f) show the time-frequency distributions of the test signal. Comparing with Figure 1(c) and Figure 1(e)-(f), we reach the conclusion that the STFRFT has higher timefrequency resolutions than STFT does. Figure 1(d) is the time-frequency distributions (TFDs) of STFRFT with the optimal order and arbitrary length of window width. Figure 1(e) is the TFDs of STFRFT with the optimal window width and arbitrary FrFT transform order. Comparing Figure 1(f) with Figure1(d)-(e), we find that the ASTFRFT has higher resolutions than STFRFT. In order to evaluate the different effects of different TFDs with quantitative methods, the energy densities of different TFDs are calculated. The TFDs are normalized by equation (9) firstly, then the energy density is calculated by equation (8). The energy density of Fig.1(c)-(f) are $3.23 \times 10^{7}, 2.87 \times 10^{7}, 2.60 \times 10^{7}$ and $2.22 \times 10^{7}$ respectively. Since the smaller the energy density is, the higher the time-frequency distribution concentration will be, the results can be concluded that the ASTFRFT has higher resolution. The second test signal is

$$
x_{2}(t)=\cos \left(50 \cos \pi t\left(t^{2}+4 \mathrm{t}\right)\right) \exp \left(\frac{-\pi}{5} t^{2}\right)+\exp \left(i \pi\left(0.5 \mathrm{t}^{2}-2.5 \mathrm{t}\right)\right) \exp \left(\frac{-\pi}{9} t^{2}\right)
$$

a)

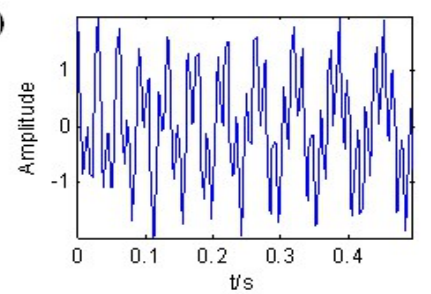

d)

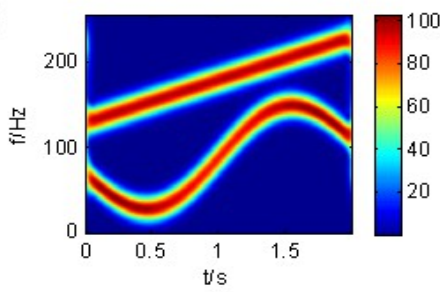

b)
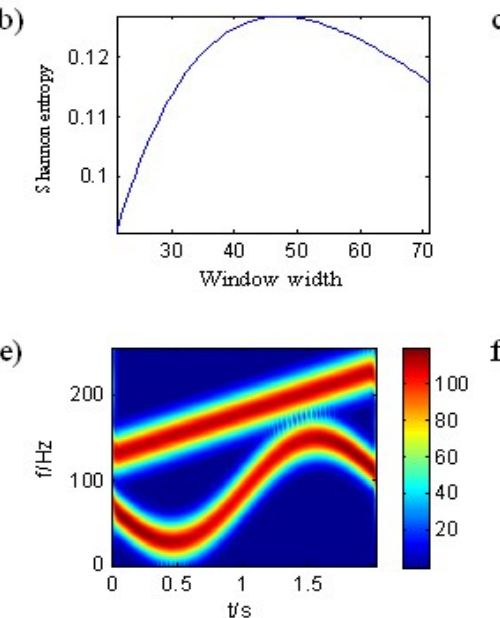

c)

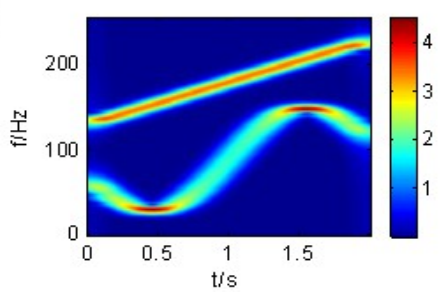

f)

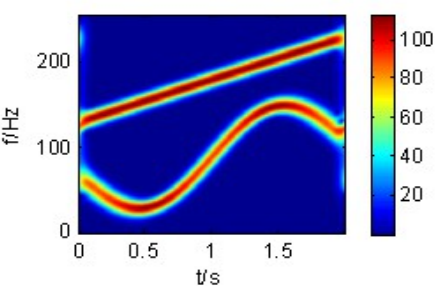

Figure 2: Non-Stationary Signal and Time-Frequency Distribution, (a) the part of test signal of $x_{2}(t)$, (b) the relationship between the Shannon entropy and the window function width, (c) STFT, (d) STFRFT with the transform order is 0.3, (e)STFRFT with 80 points window width, (f) ASTFRFT 
$x_{2}(t)$ is composed by a quadratic chirp and a sinusoidal chirp. The sampling frequency of the signal is $1 / 256 \mathrm{~Hz}$, the sampling number is 512, $t \in[0,2] \mathrm{s}$. Fig. 2(a) is part of $x_{2}(t)$, the optimal order of FrFT is 1.12 given by kurtosis maximization method, Shannon entropy with window width from 20 to 70 is shown in Fig. 2(b), the optimal window width of $x 2(t)$ is 44 . Fig. 2(c) is STFT, the window width is 128; Fig. 2(d) is the STFRFT with the transform order 0.3 and the window width is 44; Fig. 2(e) is the STFRFT with the window width 80 , and the transform order is 1.12; Fig. 2(f) is the ASTFRFT. The energy of density in Fig. 2 (c)-(f) are $6.17 \times 10^{9}, 6.87 \times 10^{9}, 9.93 \times 10^{9}$, and $5.63 \times 10^{9}$. Conclusion is that the ASTFRFT proposed in this study has higher resolution.

\section{Conclusion}

The ASTFRFT is proposed in this study. The optimal transform order is calculated by kurtosis maximization firstly, then the optimal window with is computed by Shannon entropy. Different types of non-stationary signals show that ASTFRFT has higher resolution than the traditional time-frequency. When the window width takes the optimal value, and the transform order takes other values except the optimal order, the resolution of STFRFT is lower than ASTFRFT.When transform order takes the optimal value, and the window width takes other value except the optimal value, the resolution of STFRFT is lower than ASTFRFT. Therefore ASTFRFT has higher resolution and is suitable for non-stationary signal processing.

\section{References}

[1] L. B.Almeida. The fractional Fourier transform and time-frequency representations [J]. IEEE Transactions on Signal Processing, 1994, 42(11): 3084-3091.

[2] Y. P. Chen, Z. M. Peng, ZH He, L Tian, DJ Zhang. The optimal fractional Gabor transform based on the adaptive window function and its application [J]. Applied Geophysics, 2013, 10(3): 305-313.

[3] L. Tian, Z. M. Peng. Determining the optimal order of fractional Gabor transform based on kurtosis maximization and its application [J]. Journal of Applied Geophysics, 2014, 108: 152-158.

[4] D. P. Xu , K. Guo. Fractional S transform-Part 1: Theory [J]. Applied geophysics, 2012, 9(1): 7379.

[5] X.Y. Zhang, Z. M. Peng, P.Zhang, Y. M. He and L.Tian. Spectral decomposition of seismic signal based on fractional Wigner-Ville distribution [J]. OGP,2014,49(5):839-845(in Chinese).

[6] C. Capus, K. Brown. Short-time fractional Fourier methods for the time-frequency representation of chirp signals [J]. The Journal of the Acoustical Society of America, 2003, 113(6): 3253-3263.

[7] L. J. Stanković, T.Alieva, M. J. Bastiaans. Time-frequency signal analysis based on the windowed fractional Fourier transform [J]. Signal Processing, 2003, 83(11): 2459-2468.

[8] R. Tao, Y. L. Li, Y. Wang. Short-time fractional Fourier transform and its applications [J]. IEEE Transactions on Signal Processing, 2010, 58(5): 2568-2580.

[9] C. S. Pang, L. Liu, S. Tao. Time-frequency analysis method based on short-time fractional Fourier transform [J].Act Electronica Sinica , 2013, 42(2): 347-352(in Chinese).

[10] Lu G., Xiao M., Wei P. Adaptive short time fractional Fourier transform for time-frequency segmentation[J]. Electronics Letters, 2016, 52(8):615-617. 
[11] J. Zhong, Y. Huang. Time-frequency representation based on an adaptive short-time Fourier transform [J]. IEEE Transactions on Signal Processing, 2010, 58(10): 5118-5128.

[12] X. L. Du, Y. Shen,Y. Wang . Adaptive window width selection algorithm for Gabor transform based on entropy [J]. Journal of Electronics \& Information Technology, 2008, 30(10): 2291-2294(in Chinese).

[13] G. Hu, R. Li , X. Hu. Adaptive Window Width Selection Algorithm for Discrete Gabor Transform Based on Metric Function and Its Time-frequency Filtering [J]. Journal of Computational Information Systems, 2014, 10(19): 8583-8590.

[14] B. Boashash, N. A. Khan, T. Ben-Jabeur. Time-frequency features for pattern recognition using high-resolution TFDs: A tutorial review [J]. Digital Signal Processing, 2015, 40: 1-30.

[15] C. Capus ,K. Brown. Fractional Fourier transform of the Gaussian and fractional domain signal support [J]. IEE Proceedings-Vision, Image and Signal Processing, 2003, 150(2): 99-106. 